\title{
CD30 expression in extranodal natural (a) CrossMark killer/T-cell lymphoma, nasal type among 622 cases of mature T-cell and natural killer-cell lymphoma at a single institution in South China
}

\author{
Yanfen Feng ${ }^{1,2+}$, Huilan Rao ${ }^{1,2 \dagger}$, Yiyan Lei ${ }^{3}$, Yuhua Huang ${ }^{1,2}$, Fang Wang ${ }^{1,4}$, Yu Zhang ${ }^{1,2}$, Shaoyan Xi ${ }^{1,2}$,
} Qiuliang $\mathrm{Wu}^{1,2}$ and Jianyong Shao ${ }^{1,4^{*}}$

\begin{abstract}
Background: Mature T-cell and natural killer (NK)-cell lymphomas compose a heterogeneous group of non-Hodgkin lymphomas, and extranodal NK/T-cell lymphoma, nasal type (ENKTL) is an aggressive subtype with sporadic CD30 expression. However, the significance of CD30 expression in ENKTL is controversial. We aimed to classify a large cohort of patients with mature T-cell and NK-cell lymphomas according to the 2016 World Health Organization (WHO) classification guidelines and to study the association between CD30 expression and prognosis of patients with ENKTL.

Methods: We selected consecutive patients with mature T-cell and NK-cell lymphomas who attended our institution between September 1, 2009 and August 31, 2013. We classified the lymphomas according to the 2016 revision of the WHO classification of lymphoid neoplasms, analyzed the associations between CD30 expression and clinicopathologic features of ENKTL patients, and evaluated the prognostic implications of CD30 expression.

Results: We identified 622 consecutive patients with mature T-cell and NK-cell lymphomas, including 317 (51.0\%) patients with ENKTL. In addition, CD30 expression was detected in 43 (47.3\%) of a subset of 91 patients with ENKTL. No clinicopathologic features were associated with CD30 expression, and CD30 positivity showed no prognostic significance in patients with ENKTL.
\end{abstract}

Conclusions: ENKTL is the most common type of mature T-cell and NK-cell lymphoma diagnosed at our institution. CD30 is frequently expressed in ENKTL and represents a therapeutic target; however, it may not be a prognostic marker.

Keywords: Lymphoma, T cell, Natural killer cell, CD30, Epstein-Barr virus

\section{Background}

Mature T-cell and natural killer (NK)-cell lymphoma is a rare subtype of non-Hodgkin lymphoma. According to the 2008 World Health Organization (WHO) classification of tumors of hematopoietic and lymphoid tissues, the most common subtypes of non-Hodgkin lymphoma

\footnotetext{
*Correspondence: Shaojy@sysucc.org.cn

†Yanfen Feng and Huilan Rao contributed equally to this paper

${ }^{1}$ State Key Laboratory of Oncology in South China, Collaborative Innovation Center for Cancer Medicine, Sun Yat-sen University Cancer Center, Guangzhou, Guangdong 510060, P. R. China

Full list of author information is available at the end of the article
}

are peripheral T-cell lymphoma, not otherwise specified (PTCL-NOS), anaplastic large cell lymphoma (ALCL), angioimmunoblastic T-cell lymphoma (AITL), and extranodal NK/T-cell lymphoma, nasal type (ENKTL) [1]. The subtype incidence and distribution differ according to geographical region and ethnic population. The incidence of mature T-cell and NK-cell lymphoma is relatively high in Asian countries, including China, when compared with those in the United States and Europe [2]. The classification of mature T-cell and NK-cell lymphomas has been modified in the 2016 revision of the WHO classification of lymphoid neoplasms [3]. 
In general, mature T-cell and NK-cell lymphomas are aggressive tumors with a poor prognosis, and the use of systemic chemotherapy, including anthracycline, achieves complete remission in only a fraction of all cases. The 3-year overall survival (OS) and failure-free survival (FFS) rates of patients with mature T-cell and NK-cell lymphoma are approximately $52 \%$ and $32 \%$, respectively [4]. The prognosis of patients with ENKTL is also unsatisfactory, even for those who present with early-stage disease [5]. The recent availability of brentuximab vedotin, an anti-CD30 monoclonal antibody, has led to improved OS and FFS rates among patients with refractory Hodgkin lymphoma, which is characterized by strong and uniform expression of CD30 [6]. A similar benefit was observed for patients with ALCL, a T-cell lymphoma also characterized by the strong and uniform expression of CD30 in neoplastic cells [7]. We previously reported that CD30 is expressed in ENKTL [8]; however, the significance of CD30 expression in ENKTL remains unclear.

Thus, we reviewed clinical records of a large cohort of ENKTL patients and analyzed the prognostic implications of CD30 expression. In addition, we also investigated the subtype distribution and incidence of mature T-cell and NK-cell lymphomas according to the 2016 WHO classification system in a large cancer center in South China.

\section{Methods}

\section{Patient selection}

We searched for all cases of mature T-cell and NK-cell lymphomas diagnosed in the Department of Pathology at the Sun Yat-sen University Cancer Center (Guangzhou, China) between September 1, 2009 and August 31, 2013. Diagnoses were based on the criteria of 2016 revision of WHO Classification of Lymphoid Neoplasms [3]. A series of ENKTL patients who were treated in the Department of Medicine at the Sun Yat-sen University Cancer Center during the same period were retrospectively selected to further investigate their clinical features and CD30 expression levels. The selection criteria were as follows: (1) confirmed diagnosis by pathologists, and (2) availability of complete follow-up records and pathologic materials. Patients with concomitant malignant neoplasms were excluded. Patient records were reviewed, and clinical data regarding age, gender, symptoms at presentation, site of involvement, plasma levels of Epstein-Barr virus (EBV)-DNA determined using quantitative polymerase chain reaction (Q-PCR), clinical stage, response to therapy, and status at last followup were extracted. The Institutional Review Board at the Sun Yat-sen University Cancer Center approved this study.

\section{Patient follow-up}

All patients were followed up every 6 months until May 2015. Disease progression and recurrence were diagnosed based on clinical examination, imaging assessments, and pathologic examination. OS was measured from the initiation of treatment to either the last followup or death from any cause. Progression-free survival (PFS) was measured from the initiation of treatment to the first indications of disease progression or relapse, death from any cause, or the last follow-up.

\section{Histopathologic and immunohistochemical analysis}

All the diagnoses were confirmed by seven senior pathologists from the Department of Pathology at the Sun Yatsen University Cancer Center after reviewing hematoxylin and eosin-stained tissue sections, immunohistochemistry (IHC) results, and other ancillary materials (including clinical records). A diagnostic consensus was made when the preliminary review by individual pathologists reached inconsistent diagnoses. IHC was performed on sections of 4- $\mu \mathrm{m}$ thickness. To determine the immunophenotype, IHC examination and in situ hybridization for Epstein-Barr virus-encoded small RNAs (EBERs) were performed. The main primary antibodies and antigen retrieval methods for each antibody are shown in Table 1. Appropriate positive and negative controls were included for each antibody.

The EBV Probe In Situ Hybridization Kit (DIG-AP, A300K.9901, PanPath Company, Amsterdam, the Netherlands) was used to detect EBERs according to the following procedure: (1) deparaffinization and rehydration of the paraffin sections using xylene and a series of graded ethanol solutions, respectively; (2) pretreatment of samples with $0.4 \%$ pepsin for $10 \mathrm{~min}$; (3) hybridization with digoxigenin-conjugated EBV probes at $37^{\circ} \mathrm{C}$ for $3 \mathrm{~h}$; (4) signal detection using peroxidase-conjugated antidigoxigenin antibody and 3,3'-diaminobenzidine (DAB); and (5) counterstaining of sections with a hematoxylin solution. The positive signals were brownish-yellow in color and localized within the nuclei.

CD30 was detected using a ready-to-use antibody supplied by Zymed Laboratories (Zymed-08-155, Thermo Fisher Scientific Company, South San Francisco, CA, USA). IHC was performed on sections of 4- $\mu \mathrm{m}$ thickness. Heat-induced antigen retrieval was performed using a citrate acid buffer ( $\mathrm{pH}$ 6.0) in a stainless steel high-pressure cooker for $10 \mathrm{~min}$, with the pressure set to approximately $103 \mathrm{kPa}$. The antibody was detected using DAB on a DAKO EnVision system (Dako, Carpinteria, CA, USA) with hematoxylin as a counterstain. CD30 expression was semi-quantitated in positive tumor cells. A total of 100 tumor cells in two randomly selected high-power fields were counted, and the average percentage of positive cells was calculated as the rate of CD30 expression. CD30 
Table 1 Primary antibodies and conditions used for immunohistochemical staining

\begin{tabular}{|c|c|c|c|}
\hline Primary antibody & Manufacturer (Cat. No.) & Pretreatment & Working dilution \\
\hline Mouse anti-CD20 monoclonal antibody & Zymed, San Diego, CA, USA (18-0155) & HP CB (pH 6.0) & $1: 100$ \\
\hline Mouse anti-CD79A monoclonal antibody & Novocastra, New Castle, Tyne and Wear, UK (RTU-CD79a-192) & HP CB (pH 6.0) & Ready to use \\
\hline Mouse anti-CD3 $\varepsilon$ monoclonal antibody & Novocastra, New Castle, Tyne and Wear, UK (RTU-CD3-PS1) & HP CB (pH 6.0) & Ready to use \\
\hline Mouse anti-CD45RO monoclonal antibody & Zymed, San Diego, CA, USA (08-1365) & HP CB (pH 6.0) & Ready to use \\
\hline Mouse anti-CD5 monoclonal antibody & Zymed, San Diego, CA, USA (08-1283) & HP CB (pH 6.0) & Ready to use \\
\hline Mouse anti-CD10 monoclonal antibody & Novocastra, New Castle, Tyne and Wear, UK (RTU-CD10-270) & HP CB (pH 6.0) & Ready to use \\
\hline Mouse anti-CD23 monoclonal antibody & Novocastra, New Castle, Tyne and Wear, UK (RTU-CD23-1B12) & HP CB (pH 6.0) & Ready to use \\
\hline Mouse anti-CD30 monoclonal antibody & Zymed, San Diego, CA, USA (08-0155) & HP CB (pH 6.0) & Ready to use \\
\hline Mouse anti-CD56 monoclonal antibody & Zymed, San Diego, CA, USA (08-0152) & HP CB (pH 6.0) & Ready to use \\
\hline Mouse anti-Bcl-6 monoclonal antibody & Zymed, San Diego, CA, USA (08-1426) & HP EDTA ( $\mathrm{pH} 9.0)$ & Ready to use \\
\hline Mouse anti-ALK monoclonal antibody & Zeta, Sierra Madre, CA, USA (Z2035) & HP EDTA ( $\mathrm{pH} 8.0)$ & Ready to use \\
\hline Mouse anti-Ki-67 monoclonal antibody & Zymed, San Diego, CA, USA (18-0192Z) & HP CB (pH 6.0) & $1: 50$ \\
\hline Mouse anti-TdT monoclonal antibody & Zymed, San Diego, CA, USA (18-7237) & HP EDTA (pH 9.0) & $1: 50$ \\
\hline Mouse anti-TIA-1 monoclonal antibody & Zeta, Sierra Madre, CA, USA (Z2183) & HP CB (pH 6.0) & Ready to use \\
\hline Mouse anti-Perforin monoclonal antibody & Zeta, Sierra Madre, CA, USA (Z2118) & HP CB (pH 6.0) & Ready to use \\
\hline Mouse anti-Granzyme B monoclonal antibody & Newmarket Scientific, Newmarket, Suffolk, UK (MS-1157-s) & HP CB (pH 6.0) & Ready to use \\
\hline Mouse anti-PD-1 monoclonal antibody & Zymed, San Diego, CA, USA (0381) & HP CB (pH 6.0) & $1: 100$ \\
\hline Goat anti-CXCL13 polyclonal antibody & Zymed, San Diego, CA, USA (0043) & HP CB (pH 6.0) & $1: 100$ \\
\hline Mouse anti-CD4 monoclonal antibody & Zymed, San Diego, CA, USA (0032) & HP CB (pH 6.0) & $1: 200$ \\
\hline Rabbit anti-CD8 monoclonal antibody & Zymed, San Diego, CA, USA (0043) & HP CB (pH 6.0) & $1: 100$ \\
\hline
\end{tabular}

$H P$ high pressure (boiled with buffer in a stainless steel high-pressure cooker for $10 \mathrm{~min}$, with the pressure setting at approximately $103 \mathrm{kPa}$ ), $C B$ 0.01 mol/L citrate buffer, EDTA $0.01 \mathrm{~mol} / \mathrm{L}$ ethylenediaminetetraacetic acid, Bcl-6 B-cell lymphoma-6, ALK anaplastic large cell lymphoma kinase, TdT terminal deoxynucleotidyl transferase, TIA-1 T-cell-restricted intracellular antigen-1, PD-1 programmed cell death 1, CXCL13 C-X-C motif chemokine ligand 13

positivity was defined as strong cell membrane and Golgi zone reactivity in $\geq 20 \%$ of tumor cells [9].

\section{Statistical analysis}

Categorical variables were compared using either the Chi square or Fisher's exact tests. OS and PFS were estimated using the Kaplan-Meier method. Differences were considered significant when the two-sided $P$ value was less than 0.05. All analyses were performed with SPSS 19.0 software (SPSS, Inc. Chicago, IL, USA).

\section{Results}

\section{Subtype distribution}

In total, 622 patients with mature T-cell and NK-cell lymphomas were identified during the study period. The median patient age was 47.5 years (range 2-87 years); 420 were males, and 202 were females (male-to-female ratio $=2.1: 1)$. The distribution of common histological subtypes was as follows: 317 (51.0\%) patients had ENKTL (Fig. 1a), 118 (19.0\%) had AITL (Fig. 1b), 81 (13.0\%) had PTCL-NOS (Fig. 1c), 31 (5.0\%) had anaplastic lymphoma kinase (ALK)-positive ALCL (Fig. 1d), 15 (2.4\%) had ALK-negative ALCL, and 13 (2.1\%) had primary cutaneous ALCL (Table 2).

The most common tumor site among all the patients was the upper aerodigestive tract $(n=263,42.3 \%)$, followed by the lymph nodes $(n=189,30.4 \%)$, skin $(n=70,11.3 \%)$, and gastrointestinal tract $(n=37,5.9 \%)$. Other less common sites are listed in Table 3.

Among the 317 cases of ENKTL, 240 (75.7\%) located in the upper aerodigestive tract $[164(51.6 \%)$ in the nasal cavity, $39(12.3 \%)$ in the nasopharynx, $20(6.3 \%)$ in the oropharynx, $10(3.4 \%)$ in the oral cavity, and 7 $(2.2 \%)$ in the throat], $20(6.3 \%)$ in the gastrointestinal tract, and $13(4.1 \%)$ in the skin. Less commonly involved sites included the soft tissue, orbit, pancreas, ovary, prostate, and testis. Only 5 patients with ENKTL had primary nodal involvement; nasal cavity or nasopharyngeal involvement was later discovered in 4 of these 5 patients. Among the 118 patients with AITL, 110 (92.4\%) had nodal involvement, whereas among the 81 patients with PTCL-NOS, 39 (47.6\%) had nodal involvement.

In total, 91 patients with ENKTL met the selection criteria mentioned above, and their data were collected along with available paraffin blocks and unstained slides to study the association between clinicopathologic features and CD30 expression.

\section{Clinical features of ENKTL}

The clinical features of 91 patients with ENKTL are shown in Table 4. The upper aerodigestive tract, in 

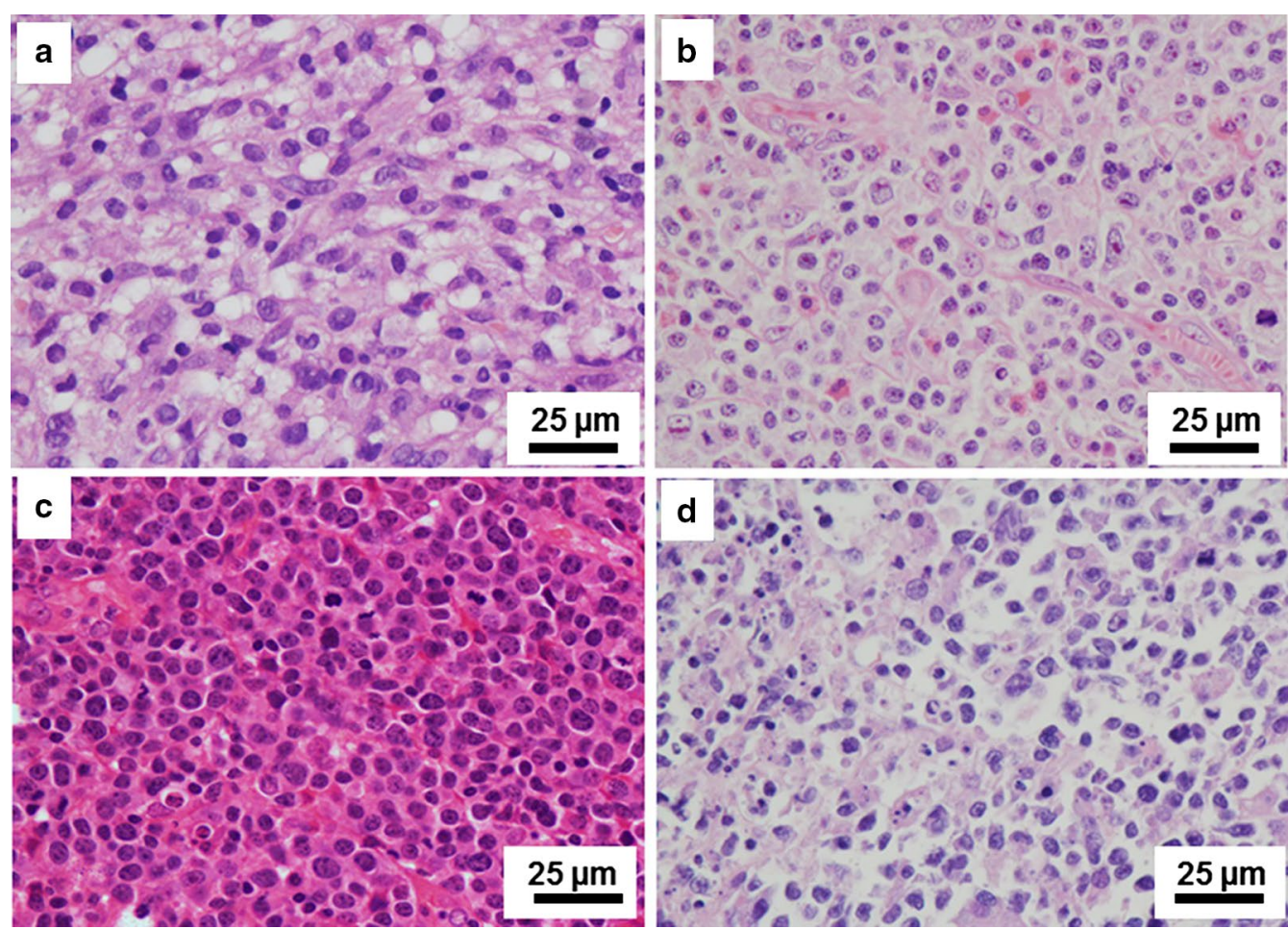

Fig. 1 Morphology of different subtypes of T-cell and natural killer (NK)-cell lymphoma (hematoxylin-eosin staining, $\times 400$ ). a Extranodal NK/T cell lymphoma, nasal type (ENKTL) shows medium-sized tumor cells with apoptotic bodies. b Angioimmunoblastic T-cell lymphoma (AITL) shows proliferation of high endothelial venules, polymorphic infiltrating cells, and cells with clear-to-pale cytoplasm. c Peripheral T-cell lymphoma, not otherwise specified (PTCL-NOS) shows medium- to large-sized polymorphic tumor cells with high mitotic figure rates. $\mathbf{d}$ Anaplastic lymphoma kinase (ALK)-positive, anaplastic large-cell lymphoma (ALCL) shows cells with horseshoe or kidney-shaped nuclei and inflammation

Table 2 Immunophenotype distribution in 622 cases of mature T-cell and NK-cell lymphoma

\begin{tabular}{|c|c|c|c|}
\hline Immunophenotype & Total [cases (\%)] & Male/female ratio & Age [years, median (range)] \\
\hline Extranodal NK/T-cell lymphoma, nasal type & $317(51.0)$ & $217 / 100$ & $43.0(10-86)$ \\
\hline Angioimmunoblastic T-cell lymphoma & $118(19.0)$ & $82 / 36$ & $63.0(36-87)$ \\
\hline Peripheral T-cell lymphoma, not otherwise specified & $81(13.0)$ & $58 / 23$ & $49.0(25-81)$ \\
\hline Anaplastic large-cell lymphoma, ALK-positive & $31(5.0)$ & $19 / 12$ & $21.0(4-69)$ \\
\hline Anaplastic large-cell lymphoma, ALK-negative & $15(2.4)$ & $10 / 5$ & $45.0(8-76)$ \\
\hline \multirow[t]{2}{*}{ Primary cutaneous, anaplastic large-cell lymphoma } & & & $48.0(24-81)$ \\
\hline & $13(2.1)$ & $4 / 9$ & $7.5(2-13)$ \\
\hline Hydroa vacciniforme-like LPD & $12(1.9)$ & $8 / 4$ & \\
\hline Subcutaneous panniculitis-like T-cell lymphoma & $12(1.9)$ & $5 / 7$ & $36.0(17-55)$ \\
\hline Monomorphic epitheliotropic intestinal T-cell lymphoma & $9(1.4)$ & $6 / 3$ & $50.0(38-82)$ \\
\hline Hepatosplenic T-cell lymphoma & $5(0.8)$ & $4 / 1$ & $37.0(29-44)$ \\
\hline Primary cutaneous CD4-positive small/medium T-cell LPD & $3(0.4)$ & $3 / 0$ & $40.0(22-60)$ \\
\hline Primary cutaneous $\gamma \delta$ T-cell lymphoma & $2(0.3)$ & $1 / 1$ & $19.0(2-36)$ \\
\hline Mycosis fungoides & $2(0.3)$ & $1 / 1$ & $29.0(18-40)$ \\
\hline Aggressive NK-cell leukemia & $2(0.3)$ & $2 / 0$ & $27.0(22-32)$ \\
\hline Total & 622 & $420 / 202$ & $47.5(2-87)$ \\
\hline
\end{tabular}

NK natural killer, $A L K$ anaplastic lymphoma kinase, $L P D$ lymphoproliferative disorder 
Table 3 Primary sites of $\mathbf{6 2 2}$ cases of mature T-cell and NKcell lymphoma

\begin{tabular}{lll}
\hline Primary site & Case(s) & Percentage (\%) \\
\hline Upper aerodigestive tract & 263 & 42.3 \\
Lymph nodes & 189 & 30.4 \\
Skin & 70 & 11.3 \\
Gastrointestinal tract & 37 & 5.9 \\
Soft tissue & 11 & 1.8 \\
Bone marrow & 10 & 1.6 \\
Orbit & 7 & 1.1 \\
Omentum & 6 & 1.0 \\
Spleen & 6 & 1.0 \\
Chest & 3 & 0.5 \\
Lung & 3 & 0.5 \\
Testicle & 3 & 0.5 \\
Bone & 2 & 0.3 \\
Liver & 2 & 0.3 \\
Ovary & 2 & 0.3 \\
Parotic gland & 2 & 0.3 \\
Prostate & 2 & 0.3 \\
Brain & 1 & 0.2 \\
Breast & 1 & 0.2 \\
Pancreas & 1 & 0.2 \\
Adrenal gland & 1 & 0.2 \\
Total & 622 & 100 \\
\hline
\end{tabular}

particular the nasal cavity, was the most common site of presentation, followed by the skin, orbit, and gastrointestinal tract. The distribution of clinical stages was as follows: stage I disease in 37 (40.7\%) patients, stage II in 34 $(37.4 \%)$ patients, stage III in $5(5.5 \%)$ patients, and stage IV in $15(16.4 \%)$ patients. Forty-one (45.1\%) patients had regional lymph node involvement. Eighty-one patients were tested for plasma EBV DNA using Q-PCR before treatment: 39 (48.1\%) were positive, with EBV DNA loads ranging from $1.07 \times 10^{3}$ to $2.49 \times 10^{6}$ copies $/ \mathrm{mL} ; 42$ (51.9\%) were negative for viral loads.

\section{Microscopic and immunohistochemical features of ENKTL}

Microscopically, ENKTL presented with necrosis, ulcerations, angiocentricity, and inflammation. The tumor cells were small, medium, or large in size and displayed irregular nuclear contours and granular chromatin.

Immunohistochemistry studies revealed that 85 (93.4\%) of the 91 cases stained positive for the NK-cell marker CD56 (Fig. 2a). All cases were positive for cytoplasmic marker CD3e and cytotoxic markers T-cell intracellular antigen-1 (TIA-1), granzyme B, and Perforin. CD5 expression was positive in 17 (20.2\%) of 84 cases. CD4 and CD8 expression was investigated in 45 cases: 40 (88.9\%) were CD4-negative and CD8-negative, 4 (8.8\%)
Table 4 Clinical features of 91 patients with extranodal natural killer (NK)/T-cell lymphoma, nasal type

\begin{tabular}{|c|c|c|}
\hline Item & Case(s) & Percentage (\%) \\
\hline \multicolumn{3}{|l|}{ Sex } \\
\hline Female & 23 & 25.3 \\
\hline Male & 68 & 74.7 \\
\hline \multicolumn{3}{|l|}{ Age (years) } \\
\hline$\leq 60$ & 81 & 89.0 \\
\hline$>60$ & 10 & 11.0 \\
\hline \multicolumn{3}{|l|}{ B symptoms } \\
\hline No & 46 & 50.5 \\
\hline Yes & 42 & 46.2 \\
\hline Unavailable & 3 & 3.3 \\
\hline \multicolumn{3}{|l|}{ Primary sites } \\
\hline Upper aerodigestive tract & 78 & 85.7 \\
\hline Skin & 5 & 5.5 \\
\hline Orbit & 3 & 3.3 \\
\hline Gastrointestinal tract & 2 & 2.2 \\
\hline Lymph node & 1 & 1.1 \\
\hline Breast & 1 & 1.1 \\
\hline Lung & 1 & 1.1 \\
\hline \multicolumn{3}{|l|}{ Ann Arbor stage } \\
\hline Stage I & 37 & 40.7 \\
\hline Stage II & 34 & 37.4 \\
\hline Stage III & 5 & 5.5 \\
\hline Stage IV & 15 & 16.4 \\
\hline \multicolumn{3}{|l|}{ Lymph node involvement } \\
\hline Yes & 41 & 45.1 \\
\hline No & 50 & 54.9 \\
\hline
\end{tabular}

were CD4-negative and CD8-positive, and 1 (2.2\%) was CD4-positive and CD8-negative. EBER was strongly and diffusely detected in the nuclei of neoplastic cells among all cases (Fig. 2b). CD30 expression was observed in 65 (71.4\%) cases. In $43(47.3 \%)$ cases, CD30 was detected in $\geq 20 \%$ of cells; based on the criteria, these cases were determined to be CD30-positive (Fig. 2c).

Fifty patients underwent bone marrow examination, and one had bone marrow involvement.

\section{Treatment and survival}

Of the 91 patients with ENKTL, 58 (63.7\%) were treated with chemoradiotherapy, 29 (31.7\%) with chemotherapy alone, $2(2.2 \%)$ with radiotherapy alone, 1 (1.1\%) with chemotherapy plus surgery (right hemicolectomy); the treatment modality was unknown for 1 patient. The most commonly used chemotherapy regimen was cyclophosphamide, doxorubicin, vincristine, and prednisone (CHOP). Thirty-one patients were treated with either pegaspargase or L-pegaspargase. Of the 71 patients with early-stage (stage I/II) disease, 56 were treated 

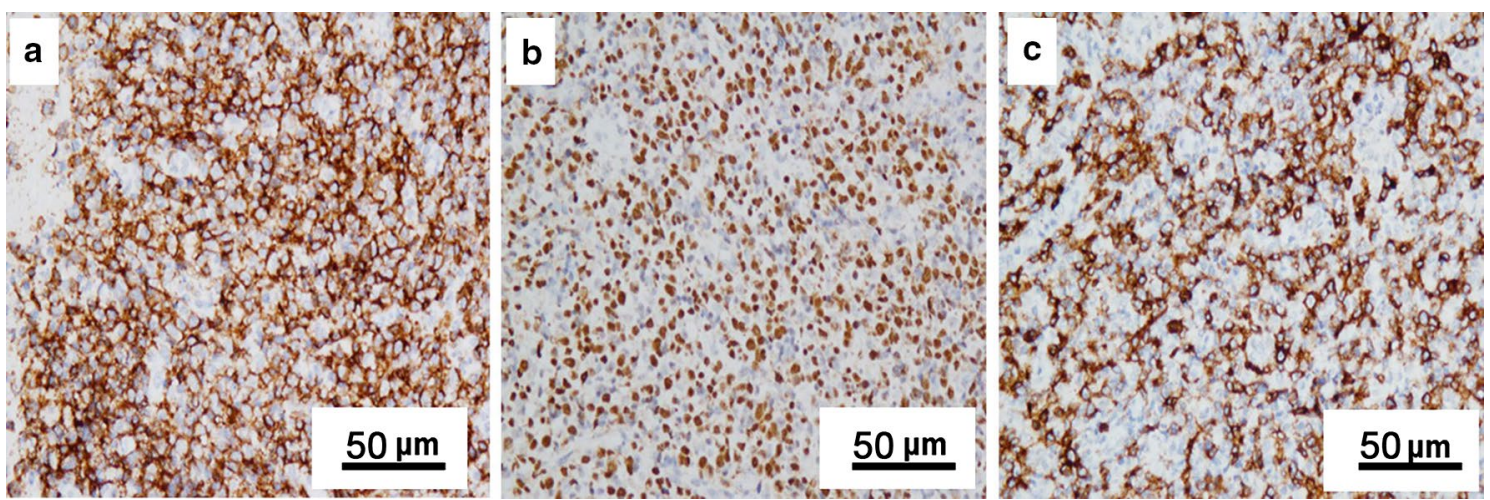

Fig. 2 Immunohistochemical staining (IHC) and in situ hybridization (ISH) staining of ENKTL. a Tumor cells show strong membranous and cytoplasmic expression of CD56 (IHC, $\times 200)$. b Tumor cells show strong nuclear expression of Epstein-Barr virus (EBV)-encoded small RNA (ISH, $\times 200)$. c Tumor cells show strong diffuse membranous and Golgi zone expression of CD30 (IHC, ×200)

with chemoradiotherapy, 13 with chemotherapy alone, and 2 with radiotherapy alone. Of the 20 patients with advanced (stage III/IV) disease, 16 were treated with chemotherapy alone, 2 with chemoradiotherapy, and 1 with chemotherapy plus surgery; the treatment modality was unknown for 1 patient. For patients who underwent radiotherapy (either radiotherapy alone or in combination with chemotherapy), the median dose was $55 \mathrm{~Gy}$ (range 40-66 Gy).

The median follow-up time was 38.5 months (range 1.1-137.1 months). The median OS was 37.8 months [95\% confidence interval (CI), 26.0-49.3 months]. The 5 -year OS rate was $66.0 \%$, with $28(30.8 \%)$ patients died during follow-up. The median PFS was 20.8 months (95\% CI, 16.5-31.2 months), and the 5-year PFS rate was
63.0\%. There was no association between CD30 positivity and either OS or PFS (both $P>0.05$ ) (Fig. 3).

There was also no association between CD30 positivity and age, sex, B symptoms, Ki-67 index, clinical stage, Ann-Arbor stage, lactate dehydrogenase level, primary site, therapeutic modality, plasma EBV-DNA status, or relapse (Table 5). However, patients who tested positive for plasma EBV-DNA had longer OS $(P=0.008)$ and PFS $(P=0.012)$ than patients negative for plasma EBV DNA (Fig. 4).

\section{Discussion}

Here we report a large cohort of 622 patients with mature T-cell and NK-cell lymphomas from a single institution in South China and outlined a basic subtype distribution
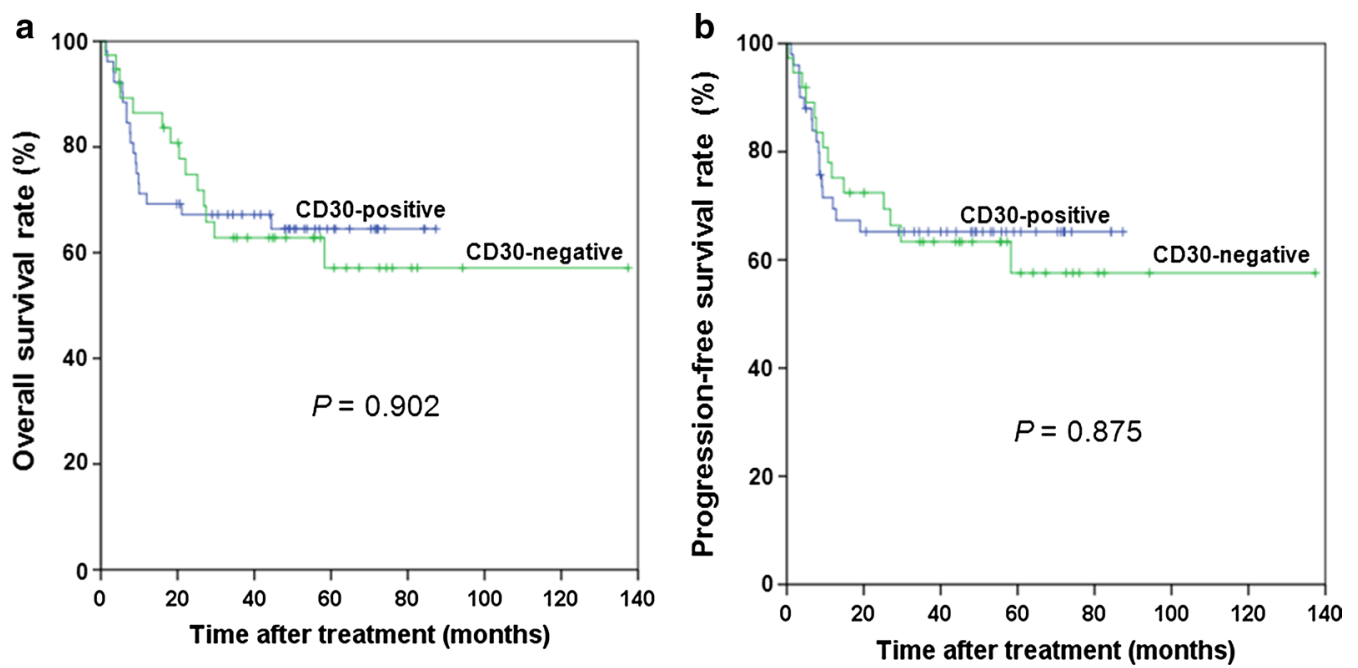

Fig. 3 Survival curves of 91 patients with ENKTL as stratified by CD30 expression. a Overall survival; b Progression-free survival 
Table 5 Association between CD30 expression and clinicopathologic features of patients with extranodal natural killer/T-cell lymphoma, nasal type

\begin{tabular}{|c|c|c|c|}
\hline \multirow[t]{2}{*}{ Variable } & \multicolumn{2}{|c|}{$\begin{array}{l}\text { CD30 expression } \\
\text { [cases }(\%)]\end{array}$} & \multirow[t]{2}{*}{$P$ value } \\
\hline & Positive & Negative & \\
\hline Total & 43 & 48 & \\
\hline Age (years) & & & 0.508 \\
\hline$\leq 60$ & $37(40.6)$ & $44(48.4)$ & \\
\hline$>60$ & $6(6.6)$ & $4(4.4)$ & \\
\hline Sex & & & 0.675 \\
\hline Male & $33(36.3)$ & $35(38.5)$ & \\
\hline Female & $10(10.9)$ & $13(14.3)$ & \\
\hline B symptoms & & & 0.627 \\
\hline Yes & $21(23.1)$ & $21(23.1)$ & \\
\hline No & $22(24.1)$ & $27(29.7)$ & \\
\hline Ki-67 index & & & 0.060 \\
\hline$\leq 50$ & $14(15.4)$ & $25(27.5)$ & \\
\hline$>50$ & $29(31.8)$ & $23(25.3)$ & \\
\hline Primary site & & & 0.720 \\
\hline Upper aerodigestive tract & $37(40.6)$ & $40(44.0)$ & \\
\hline Others & $6(6.6)$ & $8(8.8)$ & \\
\hline Ann-Arbor stage & & & 0.080 \\
\hline$|/| \mid$ & $37(40.6)$ & $34(37.4)$ & \\
\hline III/IV & $6(6.6)$ & $14(15.4)$ & \\
\hline LDH level & & & 0.894 \\
\hline Elevated & $12(13.2)$ & $14(15.4)$ & \\
\hline Normal & $31(34.0)$ & $34(37.4)$ & \\
\hline Therapeutic modality & & & 0.230 \\
\hline Chemotherapy alone & $10(10.9)$ & $19(20.9)$ & \\
\hline Chemoradiotherapy & $31(34.0)$ & $27(29.7)$ & \\
\hline Radiotherapy alone & $1(1.1)$ & $1(1.1)$ & \\
\hline Chemotherapy plus surgery & $1(1.1)$ & $0(0.0)$ & \\
\hline Unavailable & $0(0.0)$ & $1(1.1)$ & \\
\hline EBV-DNA status & & & 0.602 \\
\hline Positive & $19(20.9)$ & $20(22.0)$ & \\
\hline Negative & 18 (19.8) & $24(26.3)$ & \\
\hline Unavailable & $6(6.6)$ & $4(4.4)$ & \\
\hline Relapse & & & 0.657 \\
\hline Yes & 19 (20.9) & 19 (20.9) & \\
\hline No & $24(26.3)$ & $29(31.9)$ & \\
\hline
\end{tabular}

$L D H$ lactate dehydrogenase, EBV Epstein-Barr virus

according to the revised 2016 WHO classification system. We identified ENKTL as the most common mature T-cell and NK-cell lymphoma subtype (51.0\%) and confirmed a high frequency of CD30 expression (47.3\%) in ENKTL cases.

According to the $2008 \mathrm{WHO}$ classification of tumors of hematopoietic and lymphoid tissues, mature T-cell and NK-cell lymphomas compose a group of heterogeneous lymphomas, including ENKTL, AITL, ALCL, PTCLNOS, and other rare subtypes [1]. Recently, a revision of the WHO classification of lymphoid neoplasms and the accompanying monograph has been published [3]. This revised edition included refinements of rare types of lymphoma and introduced molecular changes in the definition of certain subtypes. Herein we classified 622 cases of mature T-cell and NK-cell lymphoma according to this revised WHO classification, which yielded new incidence data based on a large institutional study.

Within this cohort, ENKTL was the most common subtype, accounting for $51.0 \%$ of cases over a 4-year period. Similar frequencies have been reported in North China [10] and Japan [11], but these frequencies are much higher than those reported in the United States and in European countries [2]. Recently, Li et al. [12] identified the genetic risk of ENKTL based on a genome-wide association study of cases and controls from Guangdong province in South China, which indicated a genetic predisposition to ENKTL among this population.

CD30 is a member of tumor necrosis factor (TNF) receptor super-family and exerts numerous biological functions, including cell cycle arrest, apoptosis, and activation of the pro-survival transcription factor nuclear factor kappa-light-chain-enhancer of activated B cells (NF-kB). CD30 is preferentially expressed in activated $B$ cells but is also expressed in T cells and NK cells [13]. Among malignant lymphomas, CD30 is expressed in Hodgkin and Reed-Sternberg cells of Hodgkin lymphoma and in almost all neoplastic cells of ALCL. Several studies with small case series have reported CD30 expression in mature T-cell and NK-cell lymphoma, with the reported percentage of ENKTL patients that were CD30-positive ranging from $36.4 \%$ to $41 \%$ [10, 14-16]. In the current study, CD30 was expressed in $47.3 \%$ of cases, which is much higher than the rate reported in another study of mature T-cell and NK-cell lymphomas (with the exception of ALCL) [17]. CD30 has been reported to be more frequently expressed in ENKTL than in other subtypes of mature T-cell and NK-cell lymphomas, and this may be due to the concomitant presence of EBV in ENKTL patients $[1,18]$. A type II latency pattern of EBV infection has been observed in ENKTL patients, whereby tumor cells express EBERs, EBV nuclear antigen-1 (EBNA-1), latent membrane protein (LMP)-1, and LMP-2. LMP-1 is the most critical EBV element involved in cell proliferation. It induces the expression of B-cell lymphoma-2 (Bcl-2) and TNF- $\alpha$-induced protein- 3 to inhibit apoptosis. LMP-1 also up-regulates c-Myc, interleukin (IL)6 , and IL-10 to accelerate cell proliferation. LMP-1 has also been shown to up-regulate CD30 [18]; thus, LMP-1 expression may be associated with the high rate of CD30 expression among patients with ENKTL. 

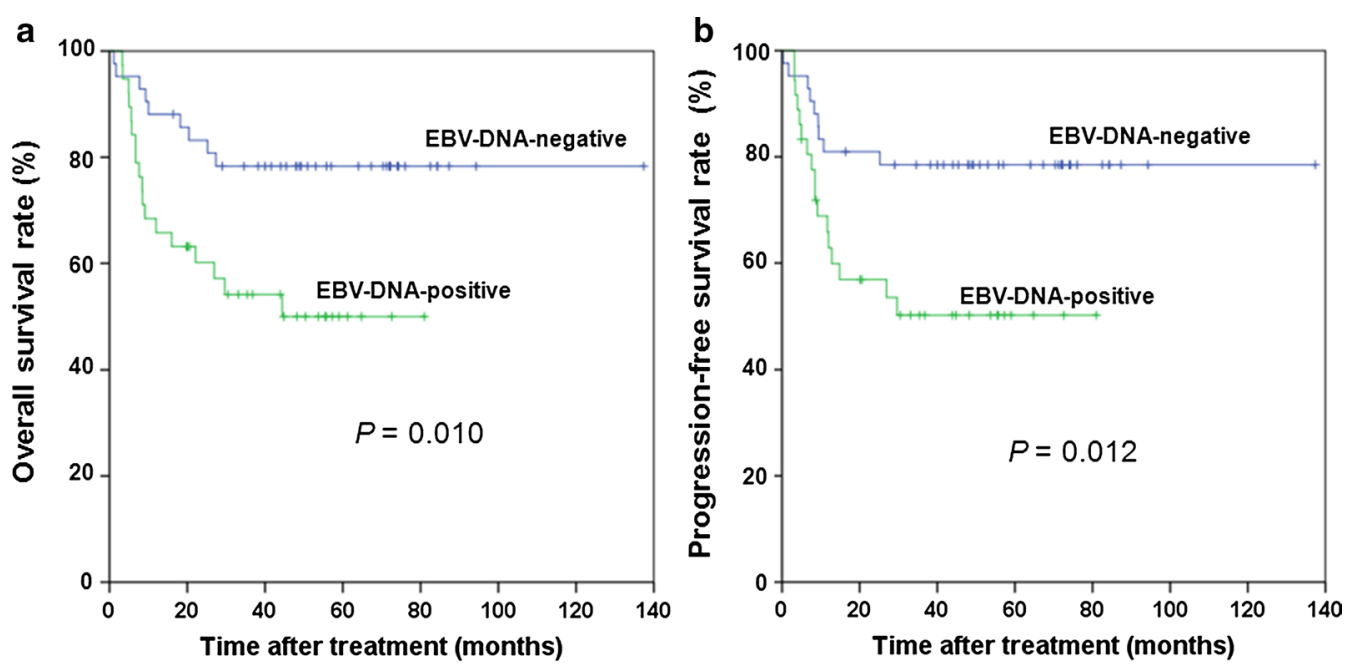

Fig. 4 Survival curves of 81 patients with ENKTL as stratified by plasma EBV-DNA status. a Overall survival; b Progression-free survival

The prognostic significance of CD30 expression in lymphomas is not well reported. Hu et al. [9] reported that CD30 expression was associated with a favorable prognosis among patients with EBV-positive diffuse large B-cell lymphoma. Bisig et al. [19] reported that patients with CD30-positive PTCL-NOS tended to have a better clinical outcome than those with CD30-negative PTCLNOS. However, the prognostic value of CD30 expression among patients with ENKTL is controversial. In one study comprising 36 patients with ENKTL, CD30 expression appeared to be associated with a favorable outcome [10]. In another study of 22 patients with ENKTL, Hong et al. [16] reported that CD30 expression was associated with a poor prognosis. Kim et al. [14] $(n=72)$ and Kuo et al. [15] $(n=22)$ revealed that there was no difference in OS among CD30-positive patients versus CD30negative patients. In our study, CD30 expression did not exhibit a prognostic significance. Since we included more cases than most (if not all) studies in the current literature, we speculate that CD30 may not be a prognostic marker for ENKTL. It is likely that a selection bias exists in our cohort because patients in our study tended to present at earlier disease stages than patients in other studies. Studies with larger referral bases are needed to determine the significance of CD30 on patient prognosis.

Although it does not appear that CD30 expression has a prognostic role in ENKTL, CD30 is a molecular target for brentuximab vedotin, an anti-CD30 monoclonal antibody, which has led to improved outcomes in patients with refractory classic Hodgkin lymphoma and ALCL [6, 20, 21]. Horwitz et al. [22] observed objective responses to brentuximab vedotin in patients with relapsed T-cell lymphomas, and these responses were seen among patients regardless of their CD30 expression status in the tumor samples. More recent researches also showed that treatment with brentuximab vedotin led to complete remission in patients with CD30-positive refractory extranodal NK/T-cell lymphoma [23] and cutaneous T-cell lymphoma [24]. Therefore, targeted therapy with an anti-CD30 antibody represents a promising management of patients with refractory ENKTL that expresses CD30. A phase 2 clinical trial is currently in processing for patients with $\mathrm{T}$-cell lymphomas (including ENKTL) to determine the effect of brentuximab vedotin on CD30-positive cases [25].

Other potential therapeutic modalities include targeting EBV antigens or using adoptive immune therapy in patients with circulating EBV DNA [26]. In our study cohort, plasma EBV DNA was detected in $48.1 \%$ of the tested cases prior to treatment initiation; the presence of EBV DNA was associated with a poorer prognosis. This result is similar to that of a previous report [27]. Plasma EBV DNA is derived from apoptotic tumor cells. It is an accurate surrogate biomarker of lymphoma load and a prognostic factor for patients treated with conventional chemotherapy [28]. However, we did not observe an association between plasma EBV DNA load and CD30 expression.

In conclusion, ENKTL is the most common subtype of mature T-cell and NK-cell lymphoma treated at our institution, a reference cancer center in South China. We identified that CD30 was expressed in a high percentage of ENKTL cases and might be a potential therapeutic molecular target for this disease; however, CD30 was insufficient as a prognostic marker. Clinical trials are needed to determine the effect of anti-CD30 therapy in patients with ENKTL that express CD30. 


\section{Authors' contributions}

YFF and HLR designed the study, and YFF drafted the manuscript. QLW contributed to the diagnoses. FW and YZ participated in the IHC studies, YHH and SYX assisted in patient selection and figure preparation. YYL performed the statistical analysis. JYS guided the whole study and revised the manuscript. All authors read and approved the final manuscript.

\section{Author details}

${ }^{1}$ State Key Laboratory of Oncology in South China, Collaborative Innovation Center for Cancer Medicine, Sun Yat-sen University Cancer Center, Guangzhou, Guangdong 510060, P. R. China. ${ }^{2}$ Department of Pathology, Sun Yat-sen University Cancer Center, Guangzhou, Guangdong 510060, P. R. China. ${ }^{3}$ Department of Thoracic Surgery, The First Affiliated Hospital of Sun Yat-sen University, Guangzhou, Guangdong 510080, P. R. China. ${ }^{4}$ Department of Molecular Diagnostics, Sun Yat-sen University Cancer Center, Guangzhou, Guangdong 510060, P. R. China.

\section{Acknowledgements}

This study was supported by the Medical Scientific Research Foundation of Guangdong Province, China (No. 2014246) and Sister Institution Network Fund of the University of Texas MD Anderson Cancer Center (to Huilan Rao).

We thank Dr. Roberto N. Miranda for editing the manuscript language.

\section{Competing interests}

The authors declare that they have no competing interests.

Received: 15 June 2016 Accepted: 5 December 2016

Published online: 10 May 2017

\section{References}

1. Swerdlow SH, Campo E, Harris NL, Jaffe ES, Pileri SA, Stein H. WHO Classification of tumors of hematopoietic and lymphoid tissues. 4th ed. Lyon: IARC Press; 2008.

2. Vose J, Armitage J, Weisenburger D. International peripheral T-cell and natural killer/T-cell lymphoma study: pathology findings and clinical outcomes. J Clin Oncol. 2008;26(25):4124-30. doi:10.1200/JCO.2008.16.4558.

3. Swerdlow SH, Campo E, Pileri SA, Harris NL, Stein H, Siebert R, et al. The 2016 revision of the World Health Organization (WHO) classification of lymphoid neoplasms. Blood. 2016;127(20):2375-90. doi:10.1182/ blood-2016-01-643569.

4. Abramson JS, Feldman T, Kroll-Desrosiers AR, Muffly LS, Winer E, Flowers $C R$, et al. Peripheral T-cell lymphomas in a large US multicenter cohort: prognostication in the modern era including impact of frontline therapy. Ann Oncol. 2014;25(11):2211-7. doi:10.1093/annonc/mdu443.

5. Niu SQ, Yang Y, Li YY, Wen G, Wang L, Li ZM, et al. Primary site and regional lymph node involvement are independent prognostic factors for earlystage extranodal nasal-type natural killer/T cell lymphoma. Chin J Cancer. 2016;35:34. doi:10.1186/s40880-016-0096-0.

6. Chen $R$, Chen B. Brentuximab vedotin for relapsed or refractory Hodgkin's lymphoma. Drug Des Devel Ther. 2015;2015(9):1729-33. doi:10.2147/ DDDT.S82007.eCollection.

7. Schirrmann T, Steinwand M, Wezler X, Ten Haaf A, Tur MK, Barth S. CD30 as a therapeutic target for lymphoma. BioDrugs. 2014;28(2):181-209. doi:10.1007/s40259-013-0068-8

8. Feng Y, Fu J, Yan S, Ling Y, Huang Y, Cai M, et al. Analysis of mature T-cell and NK-cell lymphoma with CD30 expression based on latest WHO classification. Zhonghua Bing Li Xue Za Zhi. 2014;43(8):508-11 (in Chinese)

9. Hu S, Xu-Monette ZY, Balasubramanyam A, Manyam GC, Visco C, Tzankov $A$, et al. CD30 expression defines a novel subgroup of diffuse large B-cell lymphoma with favorable prognosis and distinct gene expression signature: a report from the International DLBCL Rituximab-CHOP Consortium Program Study. Blood. 2013;121(14):2715-24. doi:10.1182/ blood-2012-10-461848.
10. Ren YL, Nong L, Zhang S, Zhao J, Zhang XM, Li T. Analysis of 142 northern Chinese patients with peripheral T/NK-Cell lymphomas: subtype distribution, clinicopathologic features, and prognosis. Am J Clin Pathol. 2012;138(3):435-47. doi:10.1309/AJCPWKJ3GPFRT7GA.

11. Niitsu N, Okamoto M, Nakamine H, Aoki S, Motomura S, Hirano M. Clinicopathologic features and outcome of Japanese patients with peripheral T-cell lymphomas. Hematol Oncol. 2008;26(3):152-8. doi:10.1002/ hon.853.

12. Li Z, Xia Y, Feng LN, Chen JR, Li HM, Cui J, et al. Genetic risk of extranodal natural killer T-cell lymphoma: a genome-wide association study. Lancet Oncol. 2016:17(9):1240-7. doi:10.1016/S1470-2045(16)30148-6.

13. Wright CW, Rumble JM, Duckett CS. CD30 activates both the canonical and alternative NF-KB pathways in anaplastic large cell lymphoma cells. J Biol Chem. 2007;282(14):10252-62.

14. Kim WY, Nam SJ, Kim S, Kim TM, Heo DS, Kim CW, et al. Prognostic implications of CD30 expression in extranodal natural killer/T-cell lymphoma according to treatment modalities. Leuk Lymphoma. 2015;56(6):1178-86. doi:10.3109/10428194.2014.974048.

15. Kuo TT, Shih LY, Tsang NM. Nasal NK/T cell lymphoma in Taiwan: a clinicopathologic study of 22 cases, with analysis of histologic subtypes, Epstein-Barr virus LMP-1 gene association, and treatment modalities. Int J Surg Pathol. 2004;12(4):375-87.

16. Hong J, Park S, Baek HL, Jung JH, Kang IG, Sym SJ, et al. Tumor cell nuclear diameter and CD30 expression as potential prognostic parameter in patients with extranodal NK/T-cell lymphoma nasal type. Int J Clin Exp Pathol. 2012;5(9):939-47.

17. Lin Z, Chen $B, X u X$, Wang $X$, Lin G. Analysis of clinical characteristics of 516 patients with non-Hodgkin's lymphoma in Shanghai area. Hematology. 2014;19(2):99-106

18. Cao M, Wang Q, Lingel A, Zhang L. Nuclear factor kB represses the expression of latent membrane protein 1 in Epstein-Barr virus transformed cells. World J Virol. 2014;3(4):22-9.

19. Bisig B, de Reyniès $A$, Bonnet $C$, Sujobert $P$, Rickman DS, Marafioti T, et al. CD30-positive peripheral T-cell lymphomas share molecular and phenotypic features. Haematologica. 2013;98(8):1250-8.

20. Molavi O, Xiong XB, Douglas D, Kneteman N, Nagata S, Pastan I, et al. Anti-CD30 antibody conjugated liposomal doxorubicin with significantly improved therapeutic efficacy against anaplastic large cell lymphoma. Biomaterials. 2013;34(34):8718-25. doi:10.1016/j.biomaterials.2013.07.068.

21. Cao ZG, Zhou HW, Peng CJ, Liu M, Du Y, Yang QM. A Chinese patient with relapsed and refractory Hodgkin lymphoma treated with brentuximab vedotin. Chin J Cancer. 2013:32(9):520-3. doi:10.5732/cjc.012.10221.

22. Horwitz SM, Advani RH, Bartlett NL, Jacobsen ED, Sharman JP, O'Connor $\mathrm{OA}$, et al. Objective responses in relapsed T-cell lymphomas with singleagent brentuximab vedotin. Blood. 2014;123(20):3095-100. doi:10.1182/ blood-2013-12-542142.

23. Kim HK, Moon SM, Moon JH, Park JE, Byeon S, et al. Complete remission in CD30-positive refractory extranodal NK/T-cell lymphoma with brentuximab vedotin. Blood Res. 2015;50(4):254-6. doi:10.5045/br.2015.50.4.254

24. Prince HM. CD30 as a target for the treatment of cutaneous T-Cell lymphoma. J Clin Oncol. 2015:33:3691-6. doi:10.1200/JCO.2015.61.9486.

25. Jagadeesh D. A phase II study of single agent brentuximab vedotin in relapsed/refractory CD30 low $(<10 \%)$ mature T cell lymphoma $(T C L)$. case comprehensive cancer center. https://Clinicaltrials.gov/show/ NCT02588651. Accessed 28 Sept 2016.

26. Tse Eric, Kwong Yok-Lam. Epstein Barr virus-associated lymphoproliferative diseases: the virus as a therapeutic target. Exp Mol Med. 2015;47:e136. doi:10.1038/emm.2014.102.

27. Au WY, Pang A, Choy C, Chim CS, Kwong YL. Quantification of circulating Epstein-Barr virus (EBV) DNA in the diagnosis and monitoring of natural killer cell and EBV-positive lymphomas in immunocompetent patients. Blood. 2004;104(1):243-9.

28. Lo YM. Quantitative analysis of Epstein-Barr virus DNA in plasma and serum: applications to tumor detection and monitoring. Ann NY Acad Sci. 2001;945:68-72. 